\title{
Risk and Management of Postoperative Urinary Retention Following Spinal Surgery
}

\author{
Kwang Suk Lee, Kyo Chul Koo, Byung Ha Chung \\ Department of Urology, Yonsei University College of Medicine, Seoul, Korea
}

Purpose: Postoperative urinary retention (POUR) is a common complication after spinal surgery. However, no clear definition of POUR currently exists, and no studies have evaluated the management of POUR. We aimed to investigate the prognostic factors for eventual POUR-free status in spinal surgery patients.

Methods: The records of patients who received a urologic consultation for POUR from January 2015 to December 2016 were reviewed. POUR-free status was defined as a voiding volume $(\mathrm{VV})>100 \mathrm{~mL}$ and a VV ratio $>50 \%$. Patients with an indwelling Foley catheter and those with any postoperative complications were excluded. The patients were divided into 2 groups according to the primary management method (Foley catheterization [FC] or intermittent catheterization [IC]).

Results: In total, 205 patients (median age, 70.6 years) were evaluated. Significant prognostic factors for eventual POUR-free status were intraoperative FC, previous spinal surgery, operative level (L3-5), lumbar fusion, and total volume (TV) at the time of POUR. Bladder training and medication did not reduce the time to POUR-free status. In patients who underwent FC, the duration of indwelling FC was a significant prognostic factor for POUR-free status. In a subanalysis, the TV ( $\geq 500 \mathrm{~mL})$ and VV ratio at the time of POUR were significant prognostic factors for POUR-free status after primary management. Among the patients who achieved a POUR-free status, 8 (6.4\%) experienced recurrent POUR. The VV ratio $(<62.0 \%)$ was the only predictor of recurrent POUR.

Conclusions: The criterion of POUR-free status is useful after spinal surgery. IC and FC were similar in their efficacy for the management of these patients.

Keywords: Postoperative Period; Spinal Surgery; Urinary Catheterization; Urinary Retention

- Research Ethics: This study was approved by the Institutional Review Board of Yonsei University College of Medicine (approval number: 2017-

0778-001). Owing to the retrospective nature of the study, written informed consent was waived.

- Conflict of Interest: No potential conflict of interest relevant to this article was reported.

\section{INTRODUCTION}

As the average life expectancy has increased, so has the number of patients who undergo spinal surgery for degenerative diseases and have lower urinary tract symptoms. In elderly patients, physiological changes, such as the replacement of muscle fibers with collagen, lead to a reduction in contractility, and changes in the nerve supply from cholinergic to nonnoradrenergic and noncholinergic fibers lead to changes in sensation [1]. Spinal surgery is related to lower urinary tract dysfunction in $38 \%-$ $60 \%$ of patients $[2,3]$. Therefore, standard guidelines for the management of urinary retention are required for patients undergoing spinal surgery.

Postoperative urinary retention (POUR), defined as im-
Corresponding author: Byung Ha Chung (iD https://orcid.org/0000-0001-9817-3660 Department of Urology, Yonsei University College of Medicine, 211 Eonjuro, Gangnam-gu, Seoul 06273, Korea

E-mail: chung646@yuhs.ac / Tel: +82-2-2019-3470 / Fax: +82-2-3462-8887

Submitted: September 25, 2017 / Accepted after revision: November 30, 2017
(9) \$ This is an Open Access article distributed under the terms of the Creative Commons Attribution Non-Commercial License (http://creativecommons.org/licenses/by-nc/4.0/) which permits unrestricted non-commercial use, distribution, and reproduction in any medium, provided the original work is properly cited. 
paired voiding after surgery, increases the duration of the hospital stay and causes pain [4]. The inadequate treatment of POUR can result in overdistention injury of the bladder, bladder detrusor muscle hypertrophy, and overactive voiding symptoms $[5,6]$. Among the conservative treatment options for POUR, Foley catheterization (FC) and intermittent catheterization (IC) are generally considered safe for short-term use in neurologic patients [7]. However, the tolerability of FC and IC varies among patients. Additionally, the indications and efficacy of each treatment and the time to removal for FC are not clear. There are few published data on pharmacologic therapy for urinary retention, as most studies have focused on prophylactic agents for POUR [8-10].

Although several studies have investigated the risk factors of POUR after lumbar spinal surgery, to the best of our knowledge, no study has explored the management of POUR in clinical practice $[11,12]$. We suggest that a voiding care protocol should be developed for the management of patients after spinal surgery. The aims of the present study were to investigate the prognostic factors of POUR-free status in patients with POUR after spinal surgery and to evaluate the efficacy of bladder training and pharmacologic therapy for treating POUR.

\section{MATERIALS AND METHODS}

Approval was obtained from the Institutional Review Board of Yonsei University College of Medicine (approval number: 20170778-001) for collecting data on all patients treated with lumbar spinal surgery at our institution. The clinical variables relevant to the study included age, sex, body mass index, intraoperative $\mathrm{FC}$, voiding volume (VV), residual volume (RV), voiding diary information, medication use (alpha-blockers), and treatment strategy.

\section{Patients}

The records of patients who received consultations at our urology department for POUR after lumbar spinal surgery from January 2015 to December 2016 were reviewed. Patients with an indwelling Foley catheter to relieve the symptom of urinary retention before spinal surgery, those taking medication for the relief of voiding symptoms before surgery, and those with urinary retention that developed as part of cauda equina syndrome in relation to a complication, such as an epidural abscess, epidural hematoma, or multiple bone fracture after trauma, were excluded.

\section{Management Protocol of POUR}

The consultation protocol in the urology department for all patients suspected of having POUR included the following evaluations: urinalysis, urine culture, uroflowmetry, and a residual urine check by IC or a bladder scan (BVI-3000 BladderScan, Verathon Inc., Bothell, WA, USA). POUR was defined as a postvoid residual urine volume (PVR) $>300 \mathrm{~mL}$ or incomplete bladder emptying [13]. FC was recommended for patients with a PVR $>700 \mathrm{~mL}$. The time to Foley catheter removal (days) was calculated as the PVR divided by 100 (for example, if the PVR was $700 \mathrm{~mL}$, the time to Foley removal was 7 days). Pharmacologic therapy was administered to treat POUR, based on the preferences of the patients' attending physicians, when POUR developed. Although bladder training is not recommended at our urology department, it was performed based upon the recommendation of the patients' attending physicians. Bladder training was performed by clamping prior to removing the urinary catheters. The clamp was released upon the desire to void or after clamping for 4-6 hours without the desire to void.

\section{Follow-up and Outcomes}

POUR-free status was defined as a VV $>100 \mathrm{~mL}$ and $\mathrm{VV}$ ratio $>50 \%$. Treatment failure was defined as (1) a change to another treatment according to the preferences of the physician and patient and (2) recurrent POUR after treatment with FC. The total volume (TV) was defined as the sum of VV. The VV ratio was calculated as VV divided by TV. The cutoff values of TV for predicting POUR-free status and the RV and VV ratio most suitable for the prediction of recurrent POUR were assessed based on the area under the receiver operating characteristic curve. The patients treated for POUR after surgery were divided into 2 groups according to the primary management method (FC vs. IC).

\section{Statistical Analysis}

Continuous variables are expressed as median (interquartile range). Categorical variables are reported as the number of occurrences and frequency. The Student t-test and Pearson chisquare test were used to statistically compare continuous and categorical variables, respectively. Cox univariate and multivariate analyses were performed to assess the prognostic factors of POUR in all spinal surgery patients. Simple and multiple logistic regressions with a forward stepwise procedure were used. All statistical comparisons were conducted with IBM SPSS Statistics ver. 23.0 (IBM Co., Armonk, NY, USA). P-values <0.05 were considered to indicate statistical significance. 


\section{RESULTS}

\section{Demographic Characteristics of Patients With POUR After Lumbar Spinal Surgery}

Table 1 shows the baseline characteristics of patients who underwent a urologic consultation for POUR after lumbar spinal surgery. A total of 205 patients (median age, 70.6 years; median operative time, 3.6 hours) were evaluated, of whom 137 (66.8\%) were operated on at the L3-5 levels. The proportions of patients with a history of spinal surgery, compression fracture, surgery at multiple levels, and lumbar fusion were $14.1 \%, 5.9 \%, 48.3 \%$, and $50.7 \%$, respectively. Medication was given to 169 patients (82.4\%) after POUR. Intraoperative FC was performed in 132 patients (85.7\%), and the catheter was removed on postopera- tive day 2. In patients with POUR, IC $(\mathrm{n}=135,65.9 \%)$ or FC $(\mathrm{n}=70,34.1 \%)$ was used as the primary management method. The FC group was younger, had a higher proportion of dura tearing, a longer operative time, a higher TV, and a longer duration of primary management and time to POUR-free status than the IC group. The failure rate of primary management over a median of 3 days was $39.0 \%$ (80 of 205). Among the patients who failed to achieve POUR-free status after primary management, 62 (77.5\%) achieved POUR-free status after a median follow-up of 7.5 days (Fig. 1).

\section{Predictors of Eventual POUR-Free Status}

After a median of 8 days for the overall treatment, the VV ratio among the patients who achieved POUR-free status was $72.5 \%$.

Table 1. Baseline characteristics of patients with urinary retention after spinal surgery

\begin{tabular}{|c|c|c|c|c|}
\hline Variable & Total $(n=205)$ & $\begin{array}{l}\text { Foley catheterization } \\
\text { group }(\mathrm{n}=70)\end{array}$ & $\begin{array}{l}\text { Intermittent catheterization } \\
\text { group }(n=135)\end{array}$ & P-value \\
\hline Age (yr) & $70.6(62.9-74.2)$ & $66.5(62.1-72.8)$ & $72.2(64.3-75.2)$ & 0.001 \\
\hline Body mass index $\left(\mathrm{kg} / \mathrm{m}^{2}\right)$ & $24.3(21.9-25.9)$ & $23.9(22.2-25.8)$ & $24.4(21.5-26.5)$ & 0.871 \\
\hline Male sex & $92(44.9)$ & $36(51.4)$ & $56(41.5)$ & 0.176 \\
\hline History of spinal surgery & $29(14.1)$ & $8(11.4)$ & $21(15.6)$ & 0.405 \\
\hline Operative level (L3-5) & $137(66.8)$ & $44(62.9)$ & $93(68.9)$ & 0.968 \\
\hline Compression fracture & $12(5.9)$ & $6(8.6)$ & $6(4.4)$ & 0.281 \\
\hline Stenosis & $112(54.6)$ & $42(60.0)$ & $70(51.9)$ & 0.269 \\
\hline Multiple level & $99(48.3)$ & $36(51.4)$ & $63(46.7)$ & 0.521 \\
\hline Lumbar fusion & $104(50.7)$ & $38(54.3)$ & $66(48.9)$ & 0.466 \\
\hline Dura tearing & $32(15.6)$ & $20(28.6)$ & $12(8.9)$ & $<0.001$ \\
\hline Operative time (hr) & $3.6(2.4-4.8)$ & $3.8(2.6-5.5)$ & $3.5(2.2-4.3)$ & $<0.001$ \\
\hline Intraoperative FC & $180(87.8)$ & $58(82.9)$ & $122(90.4)$ & 0.152 \\
\hline Time to intraoperative Foley removal (day) & $2(2-4)$ & $2(2-4)$ & $2(2-4)$ & 0.632 \\
\hline TV at POUR $(\mathrm{mL})$ & $550(450-750)$ & $750(550-900)$ & $500(400-700)$ & $<0.001$ \\
\hline VV ratio at POUR $(\%)$ & $0(0-0)$ & $0(0-9)$ & $0(0-30)$ & $<0.001$ \\
\hline Medication after POUR & $169(82.4)$ & $58(82.9)$ & $111(82.2)$ & 0.91 \\
\hline Duration of primary management (day) & $3(2-5)$ & $5(4-7)$ & $2(1-4)$ & $<0.001$ \\
\hline Failure of primary management & $80(39.0)$ & $26(37.1)$ & $54(40.0)$ & 0.692 \\
\hline Recurrent POUR after primary management & $8 / 125(6.4)$ & $3 / 44(6.8)$ & $5 / 81(6.2)$ & 0.229 \\
\hline Overall POUR-free status & $180(87.8)$ & $66(94.3)$ & $114(84.4)$ & 0.042 \\
\hline Duration of overall treatment (day) & $8(5-11)$ & $8(6-17)$ & $7(5-11)$ & 0.003 \\
\hline VV at POUR-free status (mL) & $300(200-380)$ & $300(225-387)$ & $300(200-350)$ & 0.592 \\
\hline RV at POUR-free status (mL) & $100(41-200)$ & $79(20-117)$ & $100.0(50-200)$ & 0.262 \\
\hline VV ratio POUR-free status (\%) & $72.5(56.7-88.3)$ & $77.8(64.9-93.6)$ & $65.9(52.6-83.3)$ & 0.009 \\
\hline
\end{tabular}

Values are presented as median (interquartile range) or number (\%).

FC, Foley catheterization; TV, total volume; POUR, postoperative urinary retention; VV, voiding volume; RV, residual volume. 


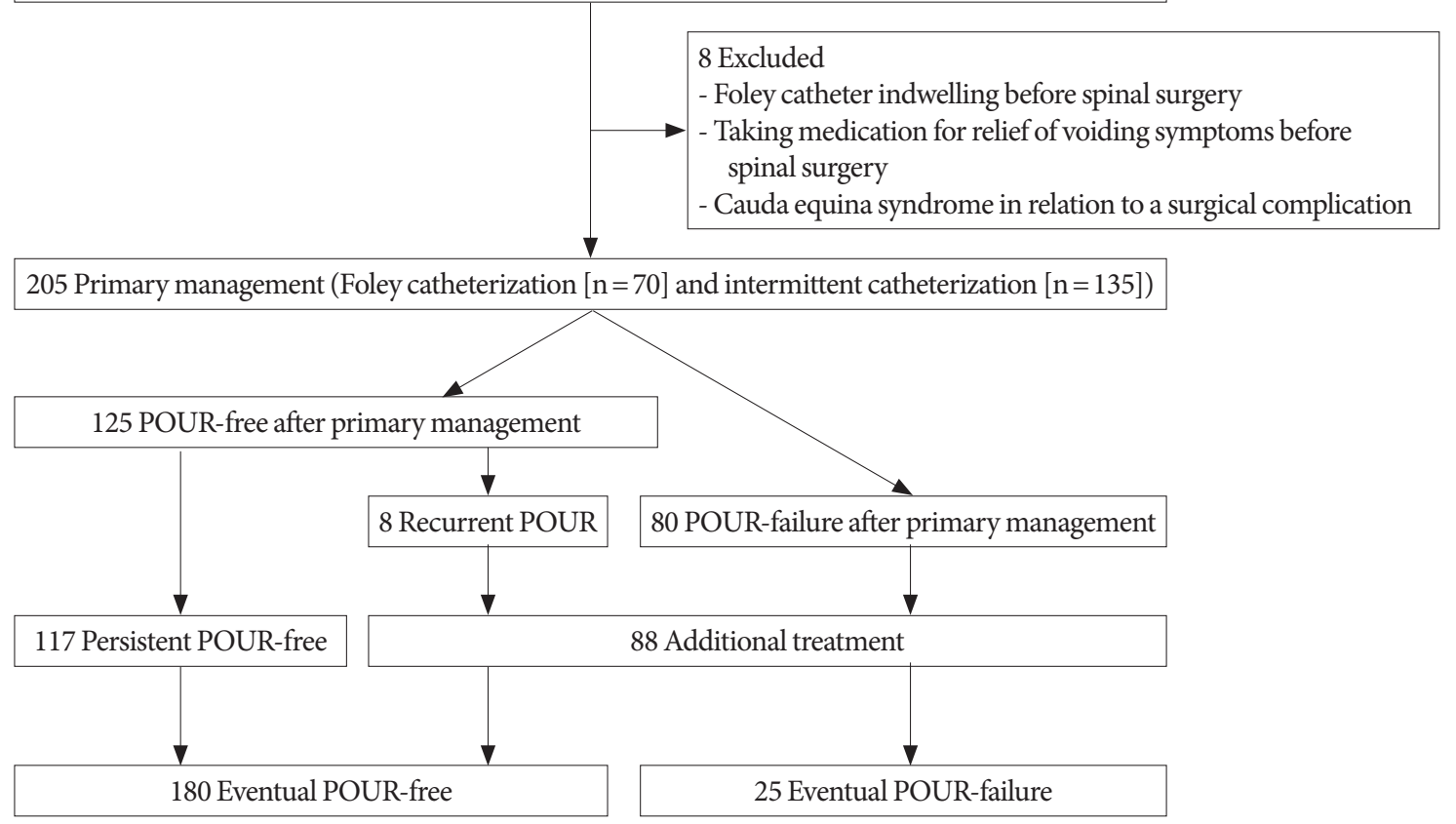

Fig. 1. Flowchart of the study selection process.

In a Cox multivariate analysis of the prognostic factors of eventual POUR-free status, intraoperative FC (hazard ratio [HR], 0.24 ; 95\% confidence interval [CI], 0.144-0.403; $\mathrm{P}<0.001$ ), previous spinal surgery $(\mathrm{HR}, 0.49 ; 95 \% \mathrm{CI}, 0.302-0.785 ; \mathrm{P}=0.003)$, operative level (L3-5) (HR, 2.00; 95\% CI, 1.437-2.778; $\mathrm{P}<0.001$ ), lumbar fusion (HR, 0.68 ; 95\% CI, 0.490-0.933; $\mathrm{P}=0.017$ ), and TV at POUR ( $\geq 500 \mathrm{~mL})(\mathrm{HR}, 0.48 ; 95 \% \mathrm{CI}, 0.346-0.661 ; \mathrm{P}<$ 0.001 ) were significant prognostic factors (Table 2 ). The primary management method for POUR (FC vs. IC) and medication use were not significant prognostic factors of eventual POURfree status.

In patients who underwent IC, intraoperative FC (HR, 0.50; 95\% CI, 0.333-0.754; $\mathrm{P}=0.001$ ), operative level (L3-5) (HR, 1.89; 95\% CI, 1.224-2.903; $\mathrm{P}=.004$ ), lumbar fusion (HR, 0.61; 95\% CI, 0.403-0.914; $\mathrm{P}=0.017)$, and TV at POUR ( $\geq 500 \mathrm{~mL})(\mathrm{HR}$, 0.28 ; 95\% CI, 0.136-0.554; $\mathrm{P}<0.001)$ were significant prognostic factors of eventual POUR-free status. In patients who underwent FC, intraoperative FC (HR, 0.21; 95\% CI, 0.088-0.0512; $\mathrm{P}=$ 0.001 ), surgery at multiple levels ( $\mathrm{HR}, 0.24 ; 95 \% \mathrm{CI}, 0.126-0.467$; $\mathrm{P}<.001$ ), medication use (HR, 0.40; 95\% CI, 0.188-0.853; $\mathrm{P}=0.018$ ), and duration of indwelling FC ( $\geq 4$ days) (HR, 2.85; 95\% CI, 1.631-4.965; $\mathrm{P}<0.001)$ were significant prognostic factors of eventual POUR-free status; bladder training was also a prognostic factor of eventual POUR-free status.
Predictors of POUR-Free Status After Primary Management In a Cox multivariate analysis of the prognostic factors of POUR-free status after primary management, a compression fracture (HR, 0.33; 95\% CI, 0.150-0.721; $\mathrm{P}=0.006)$, surgery at multiple levels (HR, 0.56; 95\% CI, 0.371-0.838; $\mathrm{P}=0.005$ ), TV at POUR ( $\geq 500 \mathrm{~mL}$ ) (HR, 0.54; 95\% CI, 0.360-0.802; $\mathrm{P}=0.002)$, VV ratio at POUR (HR, 1.03; 95\% CI, 1.024-1.042; $\mathrm{P}<0.001)$, and FC (HR, 0.39; 95\% CI, 0.250-0.606; $\mathrm{P}<0.001)$ were significant prognostic factors of POUR-free status (Table 3 ).

Subanalyses for the predictors of POUR-free status were performed according to the primary management method. In multivariate analysis, the operative level (L3-5) (HR, 3.46; 95\% CI, 1.916-6.265; $\mathrm{P}<0.001$ ), lumbar fusion (HR, 0.42; 95\% CI, 0.262-0.677; $\mathrm{P}<0.001)$, and TV at POUR $(\geq 500 \mathrm{~mL})(\mathrm{HR}$, 0.27 ; 95\% CI, $0.162-0.454$; $\mathrm{P}<0.001)$ were significant prognostic factors of POUR-free status in patients who underwent IC. Surgery at multiple levels (HR, 0.16; 95\% CI, 0.076-0.345; $\mathrm{P}<0.001)$ and the duration of indwelling FC ( $\geq 4$ days) (HR, 3.56; 95\% CI, 1.596-7.962; $\mathrm{P}=0.002$ ) were significant prognostic factors of POUR-free status in patients who underwent FC.

\section{Predictors of Recurrent POUR Among Patients With POUR- Free Status}

Among the 125 patients with POUR-free status after primary 
Table 2. Prognostic factors for overall POUR-free status in patients with urinary retention after spinal surgery

\begin{tabular}{|c|c|c|c|c|c|c|}
\hline \multirow{2}{*}{ Variable } & \multicolumn{3}{|c|}{ Univariate analysis } & \multicolumn{3}{|c|}{ Multivariate analysis } \\
\hline & HR & $95 \% \mathrm{CI}$ & P-value & HR & $95 \% \mathrm{CI}$ & P-value \\
\hline Age & 1.00 & $0.981-1.015$ & 0.811 & - & - & - \\
\hline Sex, male vs. female & 1.42 & $1.054-0.898$ & 0.021 & 1.26 & $0.929-1.704$ & 0.137 \\
\hline Intraoperative FC & 0.19 & $0.119-0.314$ & $<0.001$ & 0.24 & $0.144-0.403$ & $<0.001$ \\
\hline History of spinal surgery & 0.55 & $0.346-0.882$ & 0.013 & 0.49 & $0.302-0.785$ & 0.003 \\
\hline Operative level (L3-5) & 2.27 & $1.640-3.146$ & $<0.001$ & 2.00 & $1.437-2.778$ & $<0.001$ \\
\hline Compression fracture & 0.45 & $0.238-0.862$ & 0.016 & 0.69 & $0.350-1.373$ & 0.294 \\
\hline Stenosis & 1.09 & $0.805-1.461$ & 0.593 & - & - & - \\
\hline Multiple level & 0.53 & $0.387-0.719$ & $<0.001$ & 0.85 & $0.580-1.240$ & 0.396 \\
\hline Fixation & 0.61 & $0.453-0.823$ & 0.001 & 0.68 & $0.490-0.933$ & 0.017 \\
\hline Dura tearing & 0.83 & $0.555-1.254$ & 0.382 & - & - & - \\
\hline Operative time (hr) & 0.97 & $0.892-1.045$ & 0.38 & - & - & - \\
\hline TV at POUR $(\geq 500 \mathrm{~mL})$ & 0.57 & $0.419-0.777$ & $<0.001$ & 0.48 & $0.346-0.661$ & $<0.001$ \\
\hline VV ratio at POUR & 1.01 & $0.995-10.21$ & 0.229 & - & - & - \\
\hline Use of medication & 0.90 & $0.606-1.329$ & 0.589 & - & - & - \\
\hline Methods of management, FC vs. IC & 0.84 & $0.619-1.142$ & 0.268 & - & - & - \\
\hline
\end{tabular}

POUR, postoperative urinary retention; HR, hazard ratio; CI, confidence interval; FC, Foley catheterization; TV, total volume; VV, voiding volume; IC, intermittent catheterization.

Table 3. Prognostic factors for POUR-free status in patients who underwent primary management methods for urinary retention after spinal surgery

\begin{tabular}{|c|c|c|c|c|c|c|}
\hline \multirow{2}{*}{ Variable } & \multicolumn{3}{|c|}{ Univariate analysis } & \multicolumn{3}{|c|}{ Multivariate analysis } \\
\hline & HR & $95 \% \mathrm{CI}$ & P-value & HR & $95 \% \mathrm{CI}$ & P-value \\
\hline Age & 1.00 & $0.978-1.019$ & 0.880 & - & - & - \\
\hline Sex, male vs. female & 1.52 & $1.055-2.200$ & 0.025 & 1.12 & $0.710-1.761$ & 0.629 \\
\hline Intraoperative FC & 0.74 & $0.440-1.238$ & 0.250 & - & - & - \\
\hline History of spinal surgery & 0.77 & $0.432-1.370$ & 0.373 & - & - & - \\
\hline Operative level (L3-5) & 2.47 & $1.595-3.820$ & $<0.001$ & 1.61 & $0.971-2.661$ & 0.065 \\
\hline Compression fracture & 0.32 & $0.140-0.744$ & 0.008 & 0.33 & $0.150-0.721$ & 0.006 \\
\hline Stenosis & 1.22 & $0.851-1.759$ & 0.275 & - & - & - \\
\hline Multiple level & 0.59 & $0.409-0.851$ & 0.005 & 0.56 & $0.371-0.838$ & 0.005 \\
\hline Lumbar fusion & 0.78 & $0.541-1.112$ & 0.167 & - & - & - \\
\hline Dura tearing & 0.54 & $0.320-0.893$ & 0.017 & 1.07 & $0.578-1.962$ & 0.839 \\
\hline Operative time (hr) & 0.95 & $0.876-1.033$ & 0.234 & - & - & - \\
\hline TV at POUR $(\geq 500 \mathrm{~mL})$ & 1.00 & $0.998-0.999$ & 0.001 & 0.54 & $0.360-0.802$ & 0.002 \\
\hline VV ratio at POUR & 1.02 & $1.007-1.035$ & 0.003 & 1.03 & $1.024-1.042$ & $<0.001$ \\
\hline Use of medication & 0.79 & $0.495-1.251$ & 0.310 & - & - & - \\
\hline Methods of management, FC vs. IC & 0.36 & $0.242-0.544$ & $<0.001$ & 0.39 & $0.250-0.606$ & $<0.001$ \\
\hline
\end{tabular}

POUR, postoperative urinary retention; HR, hazard ratio; CI, confidence interval; FC, Foley catheterization; TV, total volume; VV, voiding volume; IC, intermittent catheterization. 
Table 4. Prognostic factors for recurrent POUR among patients with POUR-free status in those who underwent primary management methods for urinary retention after spinal surgery

\begin{tabular}{|c|c|c|c|c|c|c|}
\hline \multirow{2}{*}{ Variable } & \multicolumn{3}{|c|}{ Univariate analysis } & \multicolumn{3}{|c|}{ Multivariate analysis } \\
\hline & OR & $95 \% \mathrm{CI}$ & P-value & OR & $95 \% \mathrm{CI}$ & $\mathrm{P}$-value \\
\hline Age & 1.06 & $0.973-1.162$ & 0.173 & - & & - \\
\hline Sex, male vs. female & 0.39 & $0.075-2.007$ & 0.259 & - & - & - \\
\hline Operative level (L3-5) & 1.03 & $0.198-5.404$ & 0.968 & - & - & - \\
\hline Stenosis & 2.32 & $0.449-11.968$ & 0.315 & - & - & - \\
\hline Multiple level & 0.43 & $0.084-2.227$ & 0.315 & - & - & - \\
\hline Lumbar fusion & 0.89 & $0.170-4.616$ & 0.885 & - & - & - \\
\hline Operative time (hr) & 0.73 & $0.462-1.143$ & 0.167 & - & - & - \\
\hline $\mathrm{RV}$ after primary management & 1.13 & $1.008-1.019$ & 0.001 & 1.01 & $0.994-1.031$ & 0.179 \\
\hline TV after primary management & 1.08 & $1.043-1.143$ & 0.049 & 0.92 & $0.079-10.632$ & 0.946 \\
\hline VV ratio (<62.0\%) after primary management & 5.50 & $1.136-26.633$ & 0.034 & 6.31 & $1.433-27.810$ & 0.015 \\
\hline Duration of the primary treatment & 1.07 & $0.966-1.174$ & 0.204 & - & - & - \\
\hline Methods of management, FC vs. IC & 1.23 & $0.990-1.528$ & 0.062 & - & - & - \\
\hline
\end{tabular}

POUR, postoperative urinary retention; OR, odds ratio; CI, confidence interval; RV, residual volume; TV, total volume; VV, voiding volume; FC, Foley catheterization; IC, intermittent catheterization.

management, 8 (6.4\%) experienced recurrent POUR. Among the patients who experienced recurrent POUR, only 1 patient achieved eventual POUR-free status after additional treatment during the observation period. In patients with recurrent POUR, the RV, TV, and VV ratio after primary management were found to be prognostic factors in the univariate analysis. In the multivariate analysis, the $\mathrm{VV}$ ratio (<62.0\%) (odds ratio [OR], 6.31; 95\% CI, 1.433-27.810; $\mathrm{P}=0.015)$ was the only predictor of recurrent POUR. The primary management method for POUR (FC vs. IC) and medication use were not significant risk factors for recurrent POUR (Table 4).

Of the patients who underwent FC as the primary management method, 20 (28.5\%) underwent a retrograde voiding trial. However, retrograde and spontaneous voiding trials were not a predictor of recurrent POUR $(\mathrm{P}=0.605)$.

\section{DISCUSSION}

POUR is a poorly understood, yet well-recognized, postoperative complication in both men and women [13]. Although the reported incidence of POUR has varied among previous studies, approximately $20 \%$ of patients who receive general anesthesia for lumbar surgery experience POUR [14-17]. As the elderly population increases, the number of patients who develop POUR after lumbar surgery will increase. Previous studies of
POUR have focused on the investigation of preoperative/perioperative risk factors, such as age, history of benign prostatic hyperplasia, chronic constipation, and use of patient-controlled analgesia $[2,14,17,18]$. However, no clear definition of POUR currently exist, and the criteria to rule out POUR remain unclear $[18,19]$. Our findings will be used to develop a prospective POUR care protocol to be used in clinical practice.

Although very strict and aggressive criteria have been advocated to provide a maximally safe management protocol, this could lead to overtreatment in a large proportion of patients whose condition deteriorates immediately after spinal surgery. In our study, the cutoff value for the $\mathrm{VV}$ ratio was $62 \%$, and this was the only predictor of recurrent POUR. This finding was consistent with the general recommendation by urologists that clinicians should monitor patients continuously to prevent overdistention until the PVR is consistently less than one-third of the TV [20].

Altschul et al. [12] reported that intraoperative Foley placement $(\mathrm{OR}, 3.42 ; \mathrm{P}=0.004)$ was a predictor of the development of POUR in a cohort of 397 patients who underwent elective spine surgery. Our results are similar to the findings of this previous report. However, some controversy exists regarding the time to intraoperative Foley catheter removal as a prognostic factor. Although a previous study reported no significant difference in the development of POUR between patients whose 
catheter was removed on day 1 or day 3 after vaginal prolapse surgery, Chong et al. found that the incidence of POUR was significantly higher in the 1-day catheter group than in the 4-day catheter group [18, 21]. Our cohort underwent lumbar spinal surgery, and most patients routinely underwent intraoperative Foley removal on postoperative day 2. Therefore, this study identified no cutoff value of the time to intraoperative Foley removal. Regardless, no studies have evaluated the intraoperative holding period of FC in patients with POUR after spinal surgery. Our findings showed that maintaining catheter placement for $\geq 4$ days had a positive prognostic effect on patients' recovery from POUR.

In the present study, patients who underwent FC had a worse status than patients who underwent IC in terms of dura tearing, operative time, TV at POUR, and VV ratio at POUR, but no significant differences in the prognosis of eventual POUR-free status were found between those who underwent FC and IC. Some of the factors that affect the decision to perform FC are the fact that it is a bothersome and somewhat painful procedure, the possibility of long-term use, and incidence of immediate POUR after short-term indwelling catheterization. In a previous study, FC was performed when patients with POUR required more than 2 consecutive rounds of IC [12]. In this study, we identified useful parameters in clinical practice, such as a history of spinal surgery, operative level (L3-5), and lumbar fusion, as predictors of eventual POUR-free status. The distal end of the spinal cord becomes conical at the level of T12, and it is thus called the conus medullaris. This structure reaches the level of the L2 body and ends with the cauda equina, which is composed of the last nervous roots (including the ones from/to the bladder) and the filum terminale [22]. Although degenerative diseases of the spine, such as the lumbar disc, involve L4/L5 and L5/S1, this study divided patients according to whether the operative level was L1-2 or L3-5. We then identified the operative level (L3-5) as a positive prognostic factor for eventual POUR-free status. Although there was no difference in therapeutic efficacy between FC and IC, these criteria are useful for establishing a management plan in clinical settings.

The efficacy of bladder training and retrograde and spontaneous voiding trials remains controversial. Although bladder training seems to help patients restore voluntary urinary voiding, this study found that it had no significant effect on increasing the chance of a successful voiding trial in patients with POUR after spinal surgery. Our results are consistent with those of previous studies [23,24]. Geller et al. [25] assessed the diag- nostic accuracy of retrograde and spontaneous voiding trials in a randomized controlled trial in 100 patients with POUR. They reported that retrograde voiding trials, in which the bladder is filled in a retrograde manner through a catheter with $300 \mathrm{~mL}$ of sterile saline or until the patient desires voiding at maximum capacity, were more accurate for evaluating postoperative voiding dysfunction than spontaneous voiding trials. However, whether a patient underwent a retrograde or spontaneous voiding trial was not a significant factor in the prediction of recurrent POUR in our study.

Several drugs are commonly used for the management of urinary retention, including cholinergic agents, prostaglandins, alpha-blockers, and sedatives. In a recent study investigating the prophylactic effects of tamsulosin and alfuzosin, the use of alpha-blockers was associated with the prevention of POUR in men after spinal anesthesia in urologic surgery procedures [26]. However, the efficacy of pharmacologic therapy on POUR treatment remains unclear. In Korea, alpha-blockers are reimbursed for patients with POUR. Some attending physicians of patients with POUR considered the use of alpha-blockers to achieve faster bladder function recovery, to shorten the duration of hospitalization, or to reduce healthcare costs, despite the absence of high-level evidence of the efficacy of pharmacologic therapy on POUR management. This retrospective study found that medication use was not associated with eventual POURfree status in patients with POUR. However, further research into the effects of pharmacologic therapy on POUR based on a well-designed protocol is warranted.

The present study has several limitations. The indications for a urologic consultation for POUR lacked standardization, and selection bias may have been present. Although no differences in the eventual POUR-free rate were found between the FC and IC groups, FC was more frequently recommended for patients with more severe complications such as dura tearing, a longer operative time, a higher TV at POUR, and a higher VV ratio at POUR. Additionally, we were unaware of the number of patients who were missed due to not having a consultation with the urology department. Nevertheless, we believe that this effect is inherent to any retrospective study, and future studies are required for greater generalizability. Another limitation is the possibility that the inclusion of patients treated by multiple spinal surgeons at a single institution could have accounted for the heterogeneity in the results. Potential factors such as variability in surgical management and postoperative care among surgeons might limit the generalizability of our results. Finally, this 
study did not evaluate risk factors such as time to ambulation, intraoperative/perioperative anesthetic use, and past medical history (diabetes mellitus, baseline bladder function). These factors may have affected any subsequent occurrence of POUR, possibly influencing the eventual POUR-free rate. Only some patients underwent a bladder function test, such as a urodynamic study, after the incidence of POUR. However, we believe that our patients may reflect those in real-world clinical practice in terms of primary management after spinal surgery.

POUR-free status was defined as a VV $>100 \mathrm{~mL}$ and a VV ratio $>50 \%$, and this was the outcome evaluated in our efficacy analysis. There was no difference in the efficacy of POUR management between IC and FC. In patients with POUR who underwent FC, the duration of FC ( $\geq 4$ days) was a prognostic factor of POUR-free status. Bladder training and the use of alpha-blockers did not reduce the time to POUR-free status.

\section{REFERENCES}

1. Toozs-Hobson P, Parsons M, Robinson L, Robinson D. Voiding difficulties in the elderly. Post Reprod Health 2014;20:143-7.

2. Brooks ME, Moreno M, Sidi A, Braf ZF. Urologic complications after surgery on lumbosacral spine. Urology 1985;26:202-4.

3. Schwab JH, Healey JH, Rose P, Casas-Ganem J, Boland PJ. The surgical management of sacral chordomas. Spine (Phila Pa 1976) 2009;34:2700-4.

4. Baldini G, Bagry H, Aprikian A, Carli F. Postoperative urinary retention: anesthetic and perioperative considerations. Anesthesiology 2009;110:1139-57.

5. Carr LK, Webster GD. Voiding dysfunction following incontinence surgery: diagnosis and treatment with retropubic or vaginal urethrolysis. J Urol 1997;157:821-3.

6. Michelson JD, Lotke PA, Steinberg ME. Urinary-bladder management after total joint-replacement surgery. N Engl J Med 1988; 319:321-6.

7. Wyndaele JJ, Kovindha A, Madersbacher H, Radziszewski P, Ruffion A, Schurch B, et al. Neurologic urinary incontinence. Neurourol Urodyn 2010;29:159-64.

8. Lo KL, Chan MC, Wong A, Hou SM, Ng CF. Long-term outcome of patients with a successful trial without catheter, after treatment with an alpha-adrenergic receptor blocker for acute urinary retention caused by benign prostatic hyperplasia. Int Urol Nephrol 2010; 42:7-12.

9. Akkoc A, Aydin C, Topaktas R, Kartalmis M, Altin S, Isen K, et al. Prophylactic effects of alpha-blockers, Tamsulosin and Alfuzosin, on postoperative urinary retention in male patients undergoing urologic surgery under spinal anaesthesia. Int Braz J Urol 2016; 42:578-84.

10. Madani AH, Aval HB, Mokhtari G, Nasseh H, Esmaeili S, Shakiba $\mathrm{M}$, et al. Effectiveness of tamsulosin in prevention of post-operative urinary retention: a randomized double-blind placebo-controlled study. Int Braz J Urol 2014;40:30-6.

11. Lee S, Kim CH, Chung CK, Park SB, Yang SH, Kim SH, et al. Risk factor analysis for postoperative urinary retention after surgery for degenerative lumbar spinal stenosis. Spine J 2017;17:469-77.

12. Altschul D, Kobets A, Nakhla J, Jada A, Nasser R, Kinon MD, et al. Postoperative urinary retention in patients undergoing elective spinal surgery. J Neurosurg Spine 2017;26:229-34.

13. Mevcha A, Drake MJ. Etiology and management of urinary retention in women. Indian J Urol 2010;26:230-5.

14. Wright JD, Lewin SN, Deutsch I, Burke WM, Sun X, Herzog TJ. The influence of surgical volume on morbidity and mortality of radical hysterectomy for cervical cancer. Am J Obstet Gynecol 2011;205:225.e1-7.

15. Landoni F, Maneo A, Cormio G, Perego P, Milani R, Caruso O, et al. Class II versus class III radical hysterectomy in stage IB-IIA cervical cancer: a prospective randomized study. Gynecol Oncol 2001;80:3-12.

16. McLain RF, Kalfas I, Bell GR, Tetzlaff JE, Yoon HJ, Rana M. Comparison of spinal and general anesthesia in lumbar laminectomy surgery: a case-controlled analysis of 400 patients. J Neurosurg Spine 2005;2:17-22.

17. Hansen BS, Søreide E, Warland AM, Nilsen OB. Risk factors of post-operative urinary retention in hospitalised patients. Acta Anaesthesiol Scand 2011;55:545-8.

18. Chong C, Kim HS, Suh DH, Jee BC. Risk factors for urinary retention after vaginal hysterectomy for pelvic organ prolapse. Obstet Gynecol Sci 2016;59:137-43.

19. Toyonaga T, Matsushima M, Sogawa N, Jiang SF, Matsumura N, Shimojima Y, et al. Postoperative urinary retention after surgery for benign anorectal disease: potential risk factors and strategy for prevention. Int J Colorectal Dis 2006;21:676-82.

20. Geller EJ. Prevention and management of postoperative urinary retention after urogynecologic surgery. Int J Womens Health 2014; 6:829-38.

21. Alonzo-Sosa JE, Flores-Contreras JT, Paredes-Canul M. Method for transurethral catheterization for 1-3 days for pelvic floor relaxation in the postoperative period. Ginecol Obstet Mex 1997;65:4557.

22. Siracusa G, Sparacino A, Lentini VL. Neurogenic bladder and disc 
disease: a brief review. Curr Med Res Opin 2013;29:1025-31.

23. Wang LH, Tsai MF, Han CS, Huang YC, Liu HE. Is bladder training by clamping before removal necessary for short-term indwelling urinary catheter inpatient? A systematic review and meta-analysis. Asian Nurs Res (Korean Soc Nurs Sci) 2016;10:173-81.

24. Zhengyong Y, Changxiao H, Shibing Y, Caiwen W. Randomized controlled trial on the efficacy of bladder training before removing the indwelling urinary catheter in patients with acute urinary retention associated with benign prostatic hyperplasia. Scand J Urol
2014;48:400-4.

25. Geller EJ, Hankins KJ, Parnell BA, Robinson BL, Dunivan GC. Diagnostic accuracy of retrograde and spontaneous voiding trials for postoperative voiding dysfunction: a randomized controlled trial. Obstet Gynecol 2011;118:637-42.

26. Madersbacher H, Cardozo L, Chapple C, Abrams P, Toozs-Hobson $\mathrm{P}$, Young JS, et al. What are the causes and consequences of bladder overdistension? ICI-RS 2011. Neurourol Urodyn 2012;31:317-21. 